\title{
Diagnóstico laboratorial da anemia hemolítica auto-imune: características do teste manual direto do Polybrene ${ }^{\circledR}$
}

\author{
G.W. Braga, J .O. Bordin, G. Moreira J R., A. Kuroda \\ Disciplina de Hematologia e Hemoterapia, Universidade Federal de São Paulo - Escola Paulista de Medicina, São Paulo, SP.
}

\begin{abstract}
RESUMO - O teste manual direto do Polybrene ${ }^{\circledR}$ (TDP) e o teste de Coombs direto (TCD) foram utilizados para a detecção de IgG na superfície de hemácias de pacientes com diagnóstico clínico e laboratorial de anemia hemolítica auto-imune (AHAI).

Овлетіvo. Comparar a sensibilidade e especificidade do TPD e do TCD no diagnóstico da AHAI.

Método. Foram estudados 18 pacientes com diagnóstico clínico-laboratorial de AHAl. Como indivíduos controles, foram testados 20 doadores de sangue assintomáticos e 20 pacientes com anemia falciforme.

Resultados. O TCD foi positivo em 14 pacientes e negativo em quatro indivíduos, enquanto O TDP foi positivo em 17 pacientes e negativo em um indivíduo que apresentava TCD positivo devido a
\end{abstract}

\section{NTRODUÇÃO}

A anemia hemolítica auto-imune (AHAI) é caracterizada pela destruição precoce das hemácias devi do à fixação de imunogl obul inas e/ou complemento na superfície da membrana eritrocitária. O teste de Coombs direto (TCD), método habitual mente utilizado no diagnóstico da AHAI, apresenta sensibilidade limitada, desde que é positivo apenas quando o número de moléculas de IgG por glóbulo vermeIho (IgG/GV) é superior a $200^{1}$.

A detecção de auto-anticorpos eritrocitários podeser determinada por técnicas mais sensíveis que o TCD. Pode-se utilizar teste relacionado ao consumo de anticorpo que fixa complemento ${ }^{2}$, teste de formação de rosetas ${ }^{3}$, teste por radioimunoensai $0^{4}$ e teste imunoenzimático5. Recentemente, a citometria de fluxo também tem sido empregada com essa finalidade ${ }^{6}$.

Devido a complexi dade de realização e ao el evado custo dos testes mencionados, al ternativamente, neste estudo avaliou-se o comportamento do método manual direto de brometo de hexadimetrina (Pol ybrene ${ }^{\circledR}$ ) (TDP) em 18 pacientes com diagnóstico clínico de $\mathrm{AHAl}^{7}$. fixação de complemento (C 3d) nas hemácias. Todos os eluatos positivos realizados com a técnica de diclorometano revelaram anticorpos quentes com especificidade "anti-Rh". A sensibilidade do TDP (94\%) para detectar fixação de IgG in vivo foi significantemente maior $(p<0,05)$ que a do TCD (78\%), enquanto os dois testes apresentaram a mesma especificidade (100\%).

Conclusão. $O$ presente estudo permite concluir que, além de apresentar maior sensibilidade que 0 TCD, o TDP é um método rápido e de baixo custo, sendo, dessa forma, um teste auxiliar útil no diagnóstico de AHAI, especialmente nos pacientes com TCD negativo.

UNITERMOS: Anemia hemolítica auto-imune. Teste de Coombs direto. Teste manual direto do Polybrene ${ }^{\circledR}$.

\section{PACIENTES E MÉTODOS}

\section{Pacientes}

Foram estudados 18 pacientes com diagnóstico de AHAI acompanhados pela Disciplina de Hematologia e Hemoterapia da Universidade Federal de São Paulo/ Escol a Paulista de M edicina. Todos os indivíduos estudados eram caucasóides, sendo quatro $(22,2 \%)$ do sexo masculino e $14(77,8 \%)$ do feminino, com idade variando de 15 a 70 anos (média de 40,3 anos). Foram realizados exames complementares para pesquisa de doença primária em todos os pacientes. Com isso, observaram 14 pacientes com AHAI primária e quatro indivíduos com AHAI secundária a outras doenças, sendo um com lúpus eritematoso sistêmico, um com síndrome de Sjögren, um com síndrome de Evans e um com linfoma não-H odgkin.

Para control e negativo do método, O TDP também foi aplicado em 40 indivíduos sem evidência clínica de AHAI, sendo 20 doadores de sangue assintomáticos e 20 paci entes com anemia hemolíti ca her editária (anemia falciforme, HbSS).

Testes

As amostras de sangue para estudo for am col eta- 


\begin{tabular}{|c|c|c|c|c|c|c|c|c|c|}
\hline Paciente & Idade & Sexo & Diagnóstico & $\mathrm{HB} g / d L$ & RET (\%) & TCD IgG & TCD C3 & TPD & Tratamento \\
\hline AAO & 15 & $\mathrm{~F}$ & AHAI & 8,4 & 30,8 & $\mathrm{Neg}$ & Neg & Pos & Prednisona \\
\hline AGSV & 27 & $\mathrm{~F}$ & $\mathrm{AHAl}$ & 7,3 & 25,0 & Neg & Neg & Pos & Esplenectomia \\
\hline AMP & 16 & $\mathrm{~F}$ & AHAI & 7,7 & 12,5 & Pos & Pos & Pos & Prednisona \\
\hline$A C$ & 20 & $\mathrm{~F}$ & $\mathrm{AHAl}$ & 7,9 & 12,6 & Neg & Pos & Neg & Prednisona \\
\hline $\mathrm{BD}$ & 59 & $\mathrm{~F}$ & $\mathrm{AHAl}$ & 3,8 & 11,0 & Pos & Neg & Pos & Prednisona \\
\hline CPR & 70 & $\mathrm{~F}$ & $\mathrm{AHAl}$ & 5,1 & 36,0 & Pos & $\mathrm{Neg}$ & Pos & Prednisona + Esplenectomia \\
\hline $\mathrm{CQ}$ & 21 & $\mathrm{~F}$ & $\mathrm{AHAl}$ & 7,4 & 22,5 & Neg & $\mathrm{Neg}$ & Pos & Prednisona \\
\hline $\mathrm{EF}$ & 45 & M & $\mathrm{AHAl}$ & 10,1 & 3,5 & Pos & Pos & Pos & Prednisona \\
\hline JAO & 70 & M & $\mathrm{AHAl}$ & 11,0 & 8,0 & Pos & Neg & Pos & Prednisona \\
\hline LBL & 25 & $\mathrm{~F}$ & $\mathrm{AHAl}$ & 9,0 & 7,2 & Pos & Neg & Pos & Prednisona \\
\hline MJS & 58 & $\mathrm{~F}$ & $\mathrm{AHAl}$ & 8,5 & 9,4 & Pos & $\mathrm{Neg}$ & Pos & Prednisona \\
\hline MGF & 48 & $\mathrm{~F}$ & LES & 6,4 & 34,0 & Pos & Neg & Pos & Prednisona \\
\hline MU & 59 & $\mathrm{~F}$ & Sjögren & 4,3 & 31,0 & Pos & Pos & Pos & Prednisona \\
\hline PJS & 56 & M & LNH & 7,0 & 18,6 & Neg & Neg & Pos & Prednisona + Clorambucil \\
\hline RLSM & 29 & $\mathrm{~F}$ & $\mathrm{AHAl}$ & 7,5 & 18,0 & Pos & Pos & Pos & Prednisona \\
\hline SSP & 47 & $\mathrm{~F}$ & AHAI & 10,2 & 3,8 & Pos & Neg & Pos & Prednisona \\
\hline SRV & 26 & $\mathrm{~F}$ & Evans & 12,7 & 10,0 & Pos & Pos & Pos & Prednisona \\
\hline WMRF & 35 & $M$ & $\mathrm{AHAl}$ & 9,6 & 8,6 & Pos & Pos & Pos & Prednisona \\
\hline
\end{tabular}

das em tubo contendo etilenodiaminotetraacetato de sódio (EDTA) sem anti coagulante. As suspensões de hemácias a $5 \%$, preparadas com solução salina ( $\mathrm{NaCl}$ a 0,9\%), foram analisadas no dia da coleta, assim como as reações imuno-hematológicas utilizando soro. Antes dos testes, as hemácias foram lavadas seis vezes com solução salina.

Todos os pacientes foram tipados para o sistema sanguíneo $\mathrm{ABO}$, para o antígeno $\mathrm{D}\left(\mathrm{R} \mathrm{h}_{\mathrm{o}}\right)$ do sistema $\mathrm{Rh}$ e, quando necessário, para outros antígenos eritrocitários.

O TCD foi realizado mediante técnica convencional, utilizando-se soro poliespecífi co e soros monoespecíficos anti-IgG e anti-C3b, 3d ${ }^{8}$. No estudo, o TCD foi titulado até que a reação se tornasse negativa microscopicamente e, a partir dos resultados da titulação, calcul ou-se o escore do TCD ${ }^{1}$.

O eluato foi preparado pela técnica de diclorometano (DCM $)^{9}$. Para isso, as hemácias foram lavadas, adicionadas a igual volume de salina, e a ambos foi adicionado igual volume de DCM. O tubo foi agitado, centrifugado e, após retirada do DCM, aquecido a $56^{\circ} \mathrm{C}$ por dez minutos. A seguir, centrifugou-se o tubo em caçapa aquecida e a camada superior (eluato) foi imediatamente transferida para um tubo limpo. Os eluatos foram testados contra um painel dehemácias dotipo $\mathrm{O}$ previamente fenotipadas para antígenos de outros sistemas sanguíneos, incluindo cél ulas do cordão umbilical.

Realizou-se oTDP, segundo a técnica descrita por Lalezari \& J iang ${ }^{10}$, em cinco fases: 1) as hemácias do paciente foram incubadas com soro autól ogo em meio de baixa força iônica para facilitar a ligação do anticorpo à superficie celular; 2) adi cionou-se o Polybrene ${ }^{\circledR}$, policátion que promove agregação inespecífica das hemácias; 3) a seguir, foi adicionada solução de citrato de sódio, que dispersa a agregação inespecífica das hemácias não sensibilizadas, mas que não desfaz a agl utinação mediada por anticorpos que ocorreu durante a aproximação ceIular; 4) leitura macroscópica e microscópica da reação para pesquisa de aglutinados específicos secundários à reação antígeno-anticorpo; 5) nos casos de TDP microscopicamente negativos, complementou-se o teste com soro de Coombs anti-l gG ${ }^{10-12}$.

Para as reações de aglutinação foram utilizados tubos de vidro de 120 x 75mm com uma proporção de dois volumes de soro ou el uato para um volume de suspensão de hemácias a 5\%. Após a adi ção das células ao soro, a mistura foi centrifugada a 1.200 rotações por minuto (rpm), durante um minuto, ea leitura macroscópica feita por meio de delicada agitação do tubo. Os resultados negativos foram confirmados por leitura microscópica em aumento de 400 vezes. Para lavagem de células ou misturas células/soro, centrifugou-se o material a 2.400rpm durante dois minutos.

Todas as reações de aglutinação de hemácias realizadas nesse estudo, verificadas macroscopicamente e microscopicamente, foram quantificadas por mei o de sistema numérico descrito por Marsh ${ }^{13}$.

\section{RESULTADOS}

Os resultados clínicos e hematológicos dos indivíduos estudados estão relacionados na tabela 1 . 


\begin{tabular}{|lccccc|}
\hline \multicolumn{5}{|l}{$\begin{array}{l}\text { Tabela 2 } \\
\text { 18 pacientes com anemia hemolítica auto-imune }\end{array}$} \\
\hline Paciente & TCD & TCD IgG & TCD & TPD & DCM \\
& IgG & Título & C3d & & \\
AAO & Neg & 0 & Neg & Pos & Pos \\
AGSV & Neg & 0 & Neg & Pos & NR \\
AMP & Pos & $1 / 32$ & Pos & Pos & NR \\
AC & Neg & 0 & Pos & Neg & Neg \\
BD & Pos & $1 / 32$ & Neg & Pos & Pos \\
CPR & Pos & $1 / 64$ & Neg & Pos & Pos \\
CQ & Neg & 0 & Neg & Pos & Pos \\
EF & Pos & $1 / 64$ & Pos & Pos & Pos \\
JAO & Pos & $1 / 32$ & Neg & Pos & Pos \\
LBL & Pos & $1 / 32$ & Neg & Pos & Pos \\
MJS & Pos & $1 / 64$ & Neg & Pos & Pos \\
MGF & Pos & $1 / 32$ & Pos & Pos & Pos \\
MU & Pos & $1 / 1$ & Pos & Pos & NR \\
PJS & Neg & 0 & Neg & Pos & Pos \\
RLSM & Pos & $1 / 256$ & Pos & Pos & Pos \\
SSP & Pos & $1 / 256$ & Pos & Pos & Pos \\
SRV & Pos & $1 / 4$ & Pos & Pos & Pos \\
WMRF & Pos & $1 / 1$ & Pos & Pos & Pos \\
\hline
\end{tabular}

TCDIgG = teste de Coombs direto com soro anti-lgG; TCDC3d = teste de Coombs direto com soro anti-C3b, C3d; TPD = teste manual direto do Polybrene ${ }^{\circledast} ; \mathrm{DCM}=$ eluato com diclorometano.

Tabela 3 - Resultados do teste de Coombs direto (TCD) e teste manual direto do Polybrene ${ }^{\circledR}$ (TDP) aplicados em pacientes com anemia hemolítica auto-imune (AHAl), anemia falciforme (HbSS), e doadores voluntários de sangue

\begin{tabular}{|lcccc|}
\hline Grupo (N) & TCD+ & TCD- & TPD+ & TPD- \\
AHAI (18) & $14(77,8 \%)$ & $4(22,2 \%)$ & $17(94,4 \%)$ & $1(5,6 \%)$ \\
HbSS (20) & 0 & $20(100 \%)$ & 0 & $20(100 \%)$ \\
$\begin{array}{l}\text { Doadores de } \\
\text { sangue (20) }\end{array}$ & 0 & $20(100 \%)$ & 0 & $20(100 \%)$ \\
\hline
\end{tabular}

Dos 18 pacientes avaliados, $14(77,8 \%)$ apresentaram TCD positivo e quatro (22,2\%), TCD negativo. Dos indivíduos com TCD positivo, em seis $(42,9 \%)$ as hemácias foram aglutinadas com anti-IgG e antiC3, em sete $(50,0 \%)$ somente com anti-I gG, e em um $(7,1 \%)$, apenas com anti-C 3 . Somente dois pacientes apresentaram reação de fraca intensidade com padrão mi croscópico, enquanto, em 12 indivíduos, as reações foram positivas macroscopicamente.

$O$ eluato com DCM foi realizado em 15 pacientes, sendo positivo em 14 (93,3\%), até mesmo naqueles com TCD negativo, e negativo com as hemácias do indivíduo que apresentava TCD positivo somente com anti-C3. Em todos os casos, a análise imunohematológica com os eluatos revelou anticorpos ativos a $37^{\circ} \mathrm{C}$ em fase de Coombs (anticorpos quentes) que reagiram com todas as cél ulas testadas do painel, com padrão de especificidade tipo "anti-
$\mathrm{Rh}$ ", isto é, anticorpos supostamente dirigidos contra o antígeno precursor do sistema Rh.

O TDP foi positivo em 17 (94,4\%) indivíduos, incluindo os quatro pacientes com TCD negativo, porém foi negativo no paciente que apresentava TCD positivo somente com anti-C3. Mesmo nas reações de menor intensidade, o padrão de aglutinação do TDP foi sempre macroscópico, até mesmo nos indivíduos com TCD negativo ou positivo fraco. Os resultados imuno-hematológi cos dos pacientes com AHAl estão resumidos na tabela 2.

A análise laboratorial dos doadores de sangue e pacientes com anemia fal ciforme, sem evidência clínica de AHAI, revel ou TCD e TDP negativos em todos os casos. A tabela 3 resume os resultados do TCD e TDP aplicados em todos os indivíduos estudados.

A sensibilidade do TDP (94\%) para o diagnóstico de AHAI, nos 18 pacientes estudados, foi significantemente $(p<0,05)$ maior que a do TCD $(78 \%)$, enquanto os dois testes apresentaram a mesma especificidade (100\%), não sendo observado nenhum resultado falso positivo com os eritrócitos dos indivíduos do grupo controle sem AHAI.

\section{DISCUSSÃO}

Todos os eritrócitos possuem certa quantidade de IgG ligada à sua superfície. Habitualmente, indivíduos normais possuem menos de 50 moléculas de I gG por glóbulo vermelho, enquanto, geralmente, as hemácias de pacientes com AHAl estão recobertas com grande quantidade de I gG ${ }^{14}$. Além disso, alguns autores postulam que, durante o processo de envel hecimento das hemácias, ocorre um aumento do número de moléculas de I gG/GV, o que favorece o reconhecimento e fagocitose celular pelos macrófagos ${ }^{15}$.

O teste de Coombs direto tem sido empregado para demonstrar a sensibilização de hemácias in vivo auxiliando o diagnóstico de AHAI, de doença hemolítica do recém-nascido (DHRN), e a investigação de reações transfusionais. O soro de Coombs poliespecífico contém, obrigatoriamente, anticorpos com atividade anti-IgG e anti-C3d, podendo conter, também, atividade anti-C4, anti-IgM e anti-I gA. E mbora seja conveniente realizar, inicialmente, oTCD com soro pol iespecífico, énecessário que, nos testes positivos, a investigação laboratorial prossiga com reagentes monoespecíficos ${ }^{1}$.

A técnica manual do Polybrene ${ }^{\circledR}$ foi descrita, em 1980, por Lalezari \& J iang para detecção de al oanticorpos eritrocitários ${ }^{10}$. Diversos estudos confirmaram a utilidade do método como substituto do teste em salina e ao uso de enzimas em protocolos de investigação sorológica de aloanticorpos ${ }^{16,17}$. 
Além disso, proporciona mel hores resultados que a prova cruzada rápida em indivíduos sensibilizados, detectando anticorpos não diagnosticados por outros métodos, especialmente quando relacionados ao sistema $\mathrm{Rh}^{18}$.

O Polybrene ${ }^{\circledR}$ é um policátion que promove agregação lábil de hemácias, que, normalmente, é desfeita pela ação do citrato de sódio. O princípio do teste direto fundamenta-se na observação de que, quando exi ste sensi bi lização das hemácias por I gG , sua aproximação favorece a agl utinação, e o agregado não se desfaz após o tratamento com ci trato de sódio. A leitura macroscópica da reação pode ser realizada poucos minutos após a incubação dos eritrócitos com o Polybrene ${ }^{\circledR}$.

O teste manual direto foi utilizado na investigação de AHAl em pou cos estudos. Em 1982, Garratty et al. ${ }^{19}$ encontraram TDP positivo em $46,7 \%$ dos 15 pacientes com AHAI estudados. Mais recentemente, Owen \& Hows $^{7}$ observaram que o TPD foi menos sensível que o TCD em $25 \%$ dos pacientes analisados e, em $9 \%$ dos casos, foi menos sensível que o eluato. A discrepância foi atribuída à presença de alguns casos de doença de hemaglutinina fria com TCD positivo apenas com C3d. No estudo aqui apresentado, a sensibilidade do TPD foi superior à do TCD (94\% versus $78 \%, p<0,05)$, sendo positivo em quatro pacientes com TCD negativo. Em concordânci a com o estudo acima citado, O TPD foi negativo no indivíduo com hemólise devido a complemento.

Durante muitos anos, acreditou-se que caso o TCD fosse negativo não haveria IgG na superfície da hemácia; entretanto, aproximadamente 5-10\% dos pacientes com diagnóstico clínico de AHAI apresentaram TCD negativo, evidenciando que o TCD possui sensibilidade limitada ${ }^{19}$. As técnicas mais sensíveis que o TCD, empregadas para quantificar o número de moléculas de IgG ligadas à membrana eritrocitária, são de aplicação mais complexa e de custo mais el evado. Szymanski et al. descreveram um TCD automatizado capaz de detectar 100-150 moléculas de I gG/GV20, Galili et al. utilizaram formação de rosetas de hemácias ${ }^{3}$, $\mathrm{J}$ eje et al. usaram radioimunensaio ${ }^{4}$, enquanto outros autores têm quanti ficado o número de I gG/ GV com o teste imunoenzi máti co $^{21,22}$. A citometria de fluxo também pode ser empregada na análise de anticorpos eritrocitários, permitindo a quantificação de IgG/GV por meio da medida de fluorescência/GV 6 .

E m geral, as técnicas desenvol vidas para avaliar pacientes com AHAI com TCD negativo apresentam resultados satisfatórios, e o tipo de teste é escol hido em função da mel hor reproduti bilidade de resultados, facilidade de realização, menor toxicidade do material utilizado, e menor custo. Recentemente, quantificamos com mai or precisão o número de I gG/GV de pacientes com AHAI, utilizando um teste imunoenzimático (enzymelinked antigl obulin test, ELAT) ${ }^{22}$. Apesar de o ELAT possuir sensibilidade 910 vezes maior que a do TCD e não utilizar material radioativo, o teste é de real ização complexa, demorada e, portanto, de difícil implantação na rotina imuno-hematológica.

Os resultados aqui apresentados evidenciam que o TPD é um teste auxiliar rápido (menos de dez minutos), de baixo custo e de sensibilidade superior ao TCD, especialmente útil no diagnóstico de AHAl causada por IgG em pacientes com TCD negativo. Porém, assim como o TCD, O TPD não permite a identificação da especificidade do auto-anticorpo ou a subclasse da I gG que sensibiliza as hemácias. Alem disso, convém ressaltar que o TPD não detecta complemento na superfície das hemácias e pode apresentar baixa sensibilidade para detectar autoanticorpos de determinadas especificidades, tais como o auto-anti-Kell ${ }^{7}$.

E mbora as amostras de sangue para este estudo tenham sido coletadas em al guns pacientes na vigência de tratamento específico, o que poderia ter contribuído para a queda do título do auto-anticorpo, para um mesmo título de autoanti corpo em determinado paciente o TPD foi mais sensível que o TCD na detecção de sensibilização eritrocitária.

Deve-se enfatizar que o reconhecimento de hemácias pel os macrófagos não está rel acionado apenas ao número de I gG/GV, mas,também, ao arranjo das moléculas ligadas aos polipeptídeos da membrana, à subclasse da I gG que está sensibilizando as células, à quantidade de I gG livre no soro e à capacidade fagocitária do sistema histiocíticomacrofágico do indivíduo. Assim, a importância clínica do anticorpo e, conseqüentemente, a gravidade do estado hemolítico não podem ser rel aci onadas apenas aos resultados observados na análise laboratorial ${ }^{23,24}$.

\section{CONCLUSÃO}

O presente estudo permite concluir que, além de apresentar maior sensibilidade que o TCD, O TPD é um método rápido e de baixo custo, sendo, dessa forma, um testeauxiliar útil no diagnóstico de AHAI, especialmente nos pacientes com TCD negativo.

\section{SUMMARY} Laboratory diagnosis of auto-immune hemolytic
anemia: characteristics of the manual direct test
of Polybrene 
Thedirect manual Polybrene $\mathrm{T}^{\mathrm{TM}}$ test (DPT) and the direct antiglobulin tests (DAT) were employed to detect antibody sensitizing red blood cell (RCB) in patients with clinical and laboratorial findings of autoi mmune hemolytic anemia (AIHA).

PURPOSE. To compare the sensitivity and specificity of DPT and DAT in the diagnosis of AIHA.

Methods. Eighteen consecutive patients with diagnosis of AlHA were evaluated. The control group consisted of 20 normal volunteers blood donors and 20 patients with sickle cell anemia. All patients and controls were submitted to DPT and DAT. All DAT positive samples were further tested using monospeci fic reagents ( anti-I gG heavy chain and anti-C3d). Positive samples for either DPT or DAT were evaluated by el uate technique using. The dichloromethane (DCM).

RESULTS. The DAT was positive in 14 patients and negative in 4 subjects, whiletheDPT was positivein 17 patients and negativein 1 individual who had a positive DAT owing to compl ement (C3d). All positi ve eluates performed with DCM showed RBC autoantibodies with presumed "anti-Rh" specifity. The sensitivity rate of the DPT (94\%) was significantly $(p<0.05)$ higher than the sensitivity rate of DAT (78\%) to determine whether I gG was bound in vivo, but no difference was found regarding the specificity of the two tests.

CONCLUSION. 1) ThedPT is moresensitivethan the DAT in detecting IgG autoantibody on the RBCS of patients with AIHA; 2) because of its simplicity and rapidity, the DPT is a useful additional screening test for the investigation of Coombs-negative AlHA. [Rev Ass Med Brasil 1998; 44(1): 16-20.]

KEY WORDS: Autoimmune hemolytic anemia. Direct antiglobulin test. Direct manual Polybrene ${ }^{T M}$ test.

\section{REFERÊ NCIAS BIBLIOGRÁFICAS}

1. Petz LD, Garratty G. Acquired I mmune hemolytic anemias. Churchill Livingstone Inc., New York, 1980.

2. Gilliland BC, Baxter E, Evans R. Red-cell antibodies in acquired hemolytic anemia with negative antiglobulin serum tests. N Engl J Med 1971; 285: 252-6.

3. Galili U, Manny N, Izak G. EA rosette formation: a simple mean to increase sensitivity of the antiglobulin test in patients with anti red cell antibodies. Br J Haematol 1981; 47: 227-33.

4. J eje MO, Blajchman MA, Steeves K, Horsewood P, Kelton J G. Quantitation of red cell-associated IgG using an immunoradiometric assay. Transfusion 1984; 24: 473-76.

5. Leikola J , Perkins HA. Enzyme-linked antiglobulin test: an accurate and simple method to quantify red cell antibodies. Transfusion 1980; 20: 138-44.

6. Garratty G, Nance SJ . Correlation between in vivo hemolysis and the amount of red cell-bound IgG measured by flow cytometry. Transfusion 1990; 30: 617-21.

7. Owen I, Hows J. Evaluation of the manual hexadimethrine bromide (Polybrene ${ }^{\circledR}$ ) technique in the investigation of autoimmune hemolytic anemia. Transfusion 1990; 30: 814-8.

8. Walker RH. The antiglobulin test. Technical manual. American Association of Blood Banks. 10th ed. Arlington, VA, Karger, 1990; 147-57.

9. Reid ME, Sipos M. Evaluation of dichloromethane elution of routine use in a transfusion service. J Med Technol 1984; 1: $772-5$.

10. Lalezari $P$, J iang AF. The manual Polybrene ${ }^{\circledR}$ test: a simple and rapid procedure for detection of red cell antibodies. Transfusion 1980; 20: 206-11.

11. Lalezari P. The manual hexadimethrine (Polybrene $\left.{ }^{\circledR}\right)$ test: effects of serum proteins and practical applications. Transfusion 1987; 27: 295-301.

12. Malde R, Kelsall G, Knight RC. The manual low-ionic strength polybrene technique for detection of red cell antibodies. Med Lab Sci 1986; 43: 360-3.

13. Marsh WL. Scoring of hemagglutination reactions. Transfusion 1972; 12: 352-3.

14. Garratty G. Predicting the clinical significance of red cell antibodies with in vitro celular assays. Transfusion Med Rev 1990; 4: 297-312

15. Kay MMB. Mechanism of removal of senescent cells by human macrophages in situ. Proc Natl Acad Sci USA 1975; 72: 3.521-5.

16. Fisher GA. Use of the manual Polybrene ${ }^{\circledR}$ test in the routine hospital laboratory. Transfusion 1983; 23:152-4.

17. Steane EA, Steane SM, Montgomery SR, Pearson J R. A proposal for compatibility testing incorporating the manual hexadimethrine bromide (Polybrene ${ }^{\circledR}$ ) test. Transfusion 1985; 25: 540-4.

18. Lown J AG, J ohnson W, I vey J G. Eighteen months experience with a manual Polybrene ${ }^{\circledR}$ crossmatch in a large hospital transfusion laboratory. Vox Sang 1988; 55: 229-32.

19. Garratty G, Postoway N, Nance S, Brunt D. The detection of IgG on the red cells of "Coombs negative" autoimmune hemolytic anemias. Transfusion 1982; 22: 430.

20. Szymanski IO, Odgren PR, Fortier NL, Snyder LM. Red blood cell associated IgG in normal and pathologic states. Blood 1980; 55: 48-54.

21. Bodensteiner D, Brown P, Skikne B, Plapp F. The enzymelinked immunosorbent assay: accurate detection of red blood cell antibodies in autoimmune hemolytic anemia. Am J Clin Pathol 1983; 79: 182-5.

22. Bordin J O, Souza-Pinto J C, Kerbauy J. Measurement of red blood cell antibodies in autoimmune hemolytic anemia. Brazilian J Med Biol Res 1991; 24: 895-9.

23. Garratty G. The significance of IgG on the red cell surface. Transfusion Med Rev 1987; 1:47-57.

24. Zupanska B, Brojer E, Thomson EE, Merry AH, Seyfried H. Monocyte-erythrocyte interaction in autoimmune haemoIytic anaemia in relation to the number of erythrocyte-bound I gG molecules and subclass specificity of autoantibodies. Vox Sang 1987; 52: 212-8. 